EUROPEAN ORGANIZATION FOR NUCLEAR RESEARCH

European Laboratory for Particle Physics

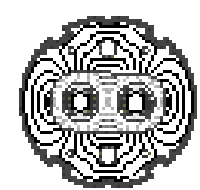

Large Hadron Collider Project

LHC Project Report 831

\title{
Measurements of the LHC Collimator Impedance with Beam in the SPS
}

\author{
G. Arduini, R. Assmann, H. Burkhardt, F. Caspers, M. Gasior, A. Grudiev, R. Jones, T. Kroyer, E. \\ Metral, S. Redaelli, G. Robert-Demolaize, F. Roncarolo, D. Schulte, R. Steinhagen, \\ J. Wenninger, F. Zimmermann \\ CERN, Geneva, Switzerland
}

\begin{abstract}
The transverse impedance of the LHC collimators will likely dominate the overall transverse impedance in the LHC at high energies and potentially limit the maximum intensity. A prototype collimator was recently tested in the SPS. Small, but significant tune shifts depending on the collimator position have been observed using different independent high resolution tune measurement methods. In addition trapped modes predicted from numerical simulation at the ends of the collimator jaws have been identified by bench measurement techniques as well as with the beam. We present a description of the measurements and an analysis of the results.
\end{abstract}

Presented at

PAC 2005, Knoxville, USA, May 16-20, 2005

CERN, CH-1211 Geneva 23,

Switzerland

Geneva, June 2005 


\title{
Measurements of the LHC Collimator Impedance with Beam in the SPS
}

\author{
H. Burkhardt, G. Arduini, R. Assmann, F. Caspers, M. Gasior, A. Grudiev, R. Jones, T. Kroyer, \\ E. Métral, S. Redaelli, G. Robert-Demolaize, F. Roncarolo, D. Schulte, R. Steinhagen, \\ J. Wenninger, F. Zimmermann, CERN, Geneva, Switzerland
}

\begin{abstract}
The transverse impedance of the LHC collimators will likely dominate the overall transverse impedance in the LHC at high energies and potentially limit the maximum intensity. A prototype collimator was recently tested in the SPS. Small, but significant tune shifts depending on the collimator position have been observed using different independent high resolution tune measurement methods. In addition trapped modes predicted from numerical simulation at the ends of the collimator jaws have been identified by bench measurement techniques as well as with the beam. We present a description of the measurements and an analysis of the results.
\end{abstract}

\section{INTRODUCTION}

Collimation in the LHC will be challenging, due to the high stored beam energy of up to $350 \mathrm{MJ}$ and relatively small beam sizes of about $0.2 \mathrm{~mm}$ r.m.s at the collimators. The collimation system will have initially $80.2 \mathrm{~m}$ long primary and $301 \mathrm{~m}$ long secondary collimators [1]. The collimator jaws will be made of carbon composite. The resistivity was measured in the laboratory and is about $\rho=10 \mu \Omega \mathrm{m}$, with good homogeneity and isotopy. The collimator impedance is expected to scale approximately as $1 / b^{3}$ where $b$ is the the distance from the beam axis. The collimators are expected to dominate the transverse LHC impedance and potentially limit the maximum beam intensity at high energy in the LHC. This motivated both theoretical [2] and experimental studies related to the collimator impedance. The possibility of coating of the jaw surface to lower the resistivity was studied, but has been abandoned.

We report here about beam based measurements, performed in the SPS on a LHC secondary collimator prototype [3]. A mounted jaw with the attached $\mathrm{Cu}$ cooling can be seen in Fig. 1.

\section{BEAM BASED IMPEDANCE MEASUREMENTS}

For a description of the principle of impedance measurements, based on the observation of the centroid motion of circulating beams in a ring, we refer to the work of Sacherer et al. [4]. The effect of the transverse impedance $Z_{\perp \text { eff }}$ (convoluted with the bunch spectrum) on the betatron motion of the bunch centroid is a complex frequency shift of

$$
\Delta \omega_{\beta}=\frac{N e c}{4 \sqrt{\pi} \omega_{\beta}(E / e) f_{\mathrm{rev}} \sigma_{t}} i\left(Z_{\perp}\right)_{\mathrm{eff}}
$$

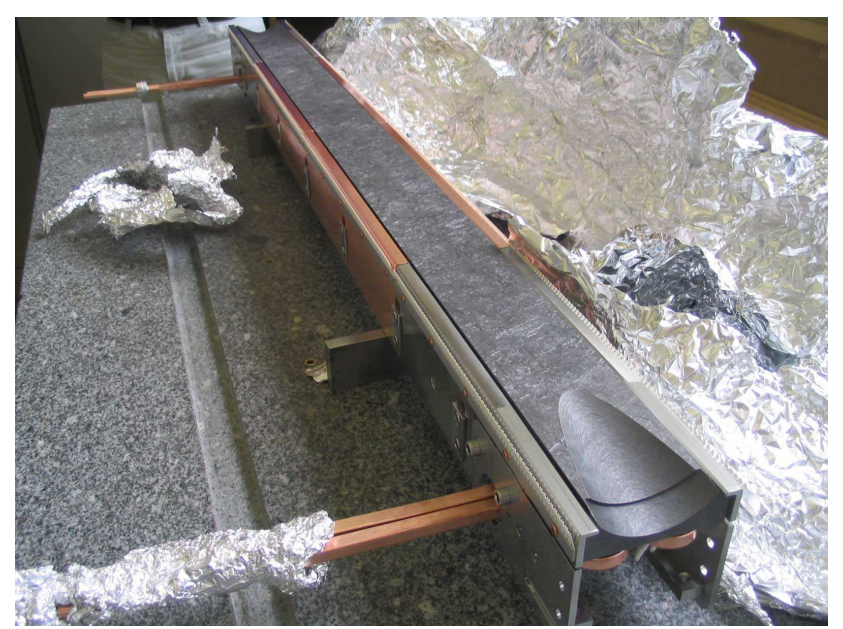

Figure 1: Secondary LHC collimator jaw. The $1 \mathrm{~m}$ long flat carbon surface and tapering at both ends, the support and $\mathrm{Cu}$ cooling is visible.

Table 1: Relevant SPS parameters

\begin{tabular}{ccc}
\hline variable & symbol & value \\
momentum & $p$ & $270 \mathrm{GeV} / \mathrm{c}$ \\
revolution frequency & $f_{\text {rev }}$ & $43375.3 \mathrm{~Hz}$ \\
betatron tunes & $Q_{x, y}$ & $\sim 26.2$ \\
momentum compaction & $\alpha_{c}$ & $1.928 \times 10^{-3}$ \\
bunch population & $N$ & $6-10 \times 10^{10}$ \\
rms bunch duration & $\sigma_{t}$ & $\sim 0.7 \mathrm{~ns}$ \\
\hline
\end{tabular}

where $N$ is the number of particles in the bunch, $E$ its energy, $f_{\text {rev }}$ the revolution frequency, $\sigma_{t}=\sigma_{z} / c$ the bunch duration and $\omega_{\beta}=2 \pi Q f_{\text {rev }}$ the betatron frequency.

We expect a scaling of the frequency shift with energy and gap (half) opening of $\Delta \omega_{\beta} \sim 1 /\left(\gamma b^{3}\right)$, where $\gamma$ is the Lorentz factor $\left(\gamma=E / m c^{2}\right)$. The measurements were done with protons in the SPS. Transverse beam sizes $\sigma$ scale with $1 / \sqrt{\gamma}$. For a collimator setting at $n_{\sigma}$, we can therefore expect that the tune shift will scale with $\sim \sqrt{\gamma}$ and be largest at highest energy. The measurements were therefore done at $270 \mathrm{GeV}$, the highest possible SPS energy in constant energy mode ("coast").

The betatron tunes in the SPS were measured independently with several instruments. We used a recently installed "Base-Band Q" (BBQ) monitor [5] and a $245 \mathrm{MHz}$ resonant strip-line pickup. These instruments were sufficiently sensitive to observe the betatron tunes without excitation. The tune measurement precision is about $10^{-5}$, with excellent agreement between the two instruments and 
mainly limited by the machine stability. Few points were in addition cross checked using the standard tune meter which requires beam excitation with a kicker. All three instruments showed small, but very significant tune changes, correlated with collimator position. Fig 2 shows an example of the spectra recorded as a function of time with the BBQ instrument.

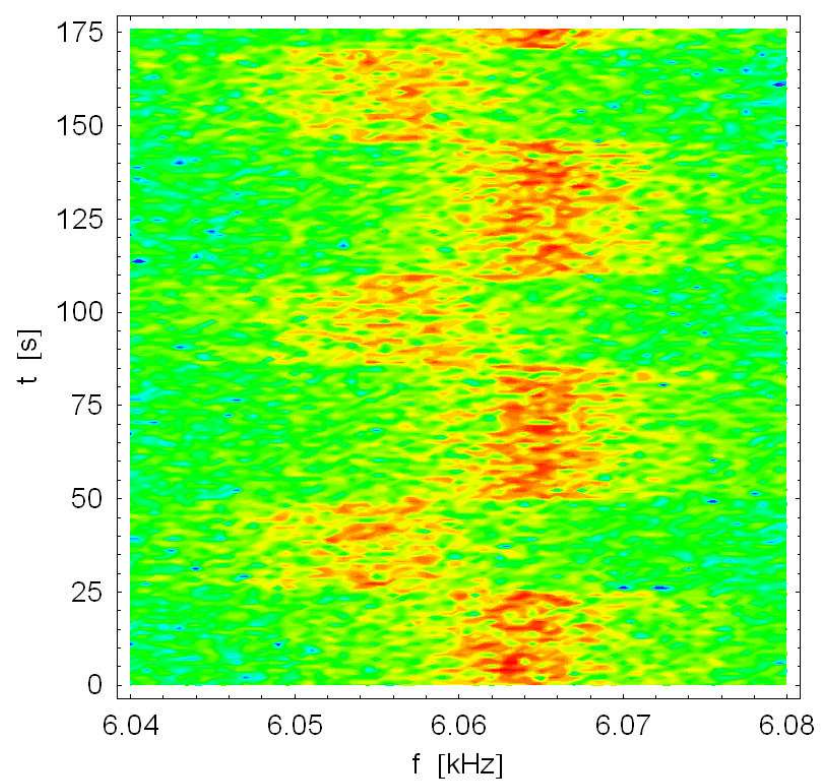

Figure 2: Spectra recorded with the BBQ in logarithmic colour coding. Green is low and red high amplitude. The periodic left right shifts correspond to closing and opening of the collimator gaps, in this case to $b=1.23 \mathrm{~mm}$. The frequency of $6.060 \mathrm{kHz}$ corresponds to a fractional tune of $q_{x}=0.1397$.

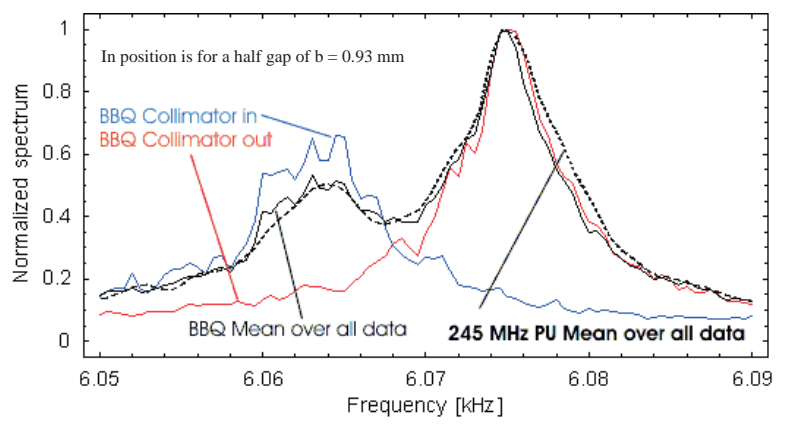

Figure 3: Comparison of time averaged spectra recorded with the BBQ and $245 \mathrm{MHz}$ pickup.

Fig 3 shows the comparison of time averaged spectra for the two instruments BBQ and the $245 \mathrm{MHz}$ pickup. Two well separated peaks, corresponding to the collimator in and out positions are clearly visible and the agreement between both instruments is excellent. It is interesting to note that both instruments recorded higher amplitudes when the collimators were open and smaller when they were closed. At present, we do not have a detailed model which would allow to predict the observed amplitudes. It is likely, that both head-tail damping and non-linear wake field effects play a role.

We recorded several series of measurements. In each case, a single bunch of typically $10^{11}$ protons was injected into the SPS and ramped to $270 \mathrm{GeV}$, with collimators retracted. Centroid bunch positions were then continuously recorded for every turn. Intensities and bunch length were monitored as well. Collimators were then closed and retracted periodically at about 30 second intervals to a first position of about $2.5 \mathrm{~mm}$ half gap opening. The same was then repeated several times, going periodically to tighter collimator settings, like several times $2 \mathrm{~mm}, 1.5 \mathrm{~mm}$, down to about $1 \mathrm{~mm}$ half gap opening which corresponds to about $2 \sigma$ with about 60 to $70 \%$ of the initial beam intensity remaining. The tune measurements and collimator movements reported here were all in the horizontal plane.

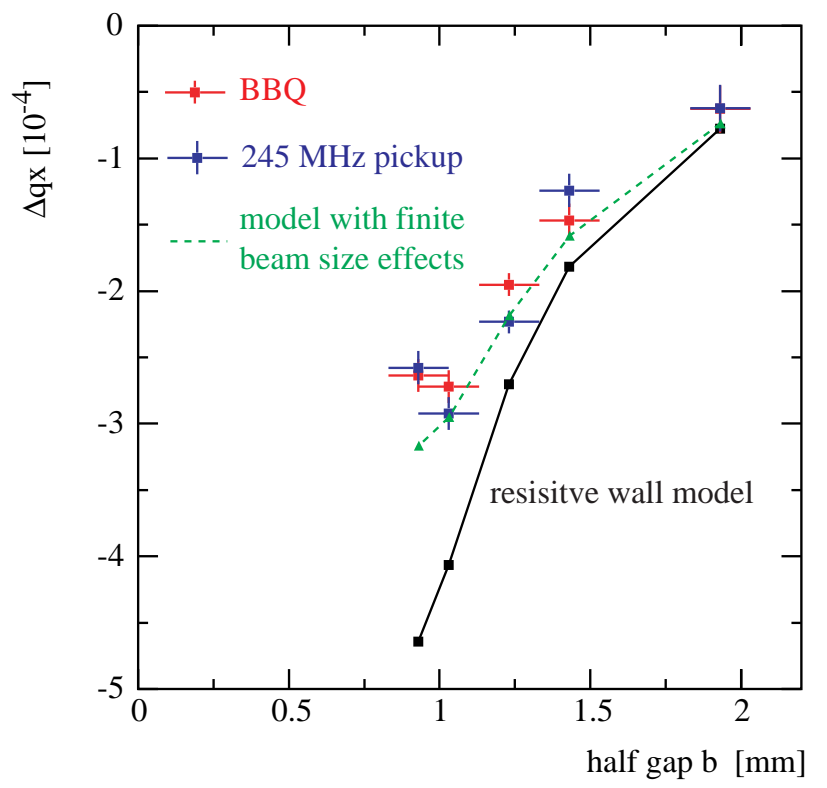

Figure 4: Comparison of measurements and predictions.

Figure 4 shows measured tune shifts between collimator in/out positions and predictions based on a resistive wall model. The tune shifts were measured independently with two instruments and the data are in good agreement. The horizontal error bars show the uncertainty in the knowledge of the gap width. The vertical errors give an estimate of the tune measurement precision and reproducibility. There is a good agreement between observed and predicted tune shifts for gap widths above $1.5 \mathrm{~mm}$. For smaller gaps, the observed tune shifts are smaller than predicted by resistive wall theory. This gives us confidence, that the predictions by the resistive wall model will be rather on the safe side.

The general resistive-wall wake-field deflection $\Delta y_{\mathrm{nl}}^{\prime}\left(x_{0}, y_{0}, x, y, \tau\right)$ for two parallel horizontal plates, including its complete nonlinear dependence on the transverse positions of drive $\left(x_{0}, y_{0}\right)$ and test particles $(x, y)$, separated by a distance $\tau$, was derived by Bane, 
Irwin and Raubenheimer [6] from the impedance formula of Piwinski [7]. Differentiating $\Delta y_{\mathrm{nl}}^{\prime}$ with respect to a vertical offset of the bunch centroid $y_{\mathrm{c}}$, the coherent tune shift including nonlinear and finite-beam size effects is obtained by integrating over the transverse distributions of both drive and source particles as

$$
\begin{gathered}
\left.\left.\Delta Q_{y}=-\frac{1}{n} \frac{\beta}{4 \pi} \int_{-\infty}^{\infty} \int_{-\infty}^{\infty} \int_{-g+2 y_{\mathrm{co}}}^{g} \int_{-g+2 y_{\mathrm{co}}}^{g}\right]_{y_{\mathrm{c}}=y_{\mathrm{co}}}\right\rangle_{\tau} \\
\left\langle\left[\frac{\partial\left(\Delta y_{\mathrm{nl}}^{\prime}\left(\tilde{x}, y_{\mathrm{c}}+\tilde{y}, x_{0}, y_{\mathrm{c}}+\tilde{y}_{0}, \tau\right)\right.}{\partial y_{0}}\right]\right. \\
\frac{e^{-\frac{y^{2}}{2 \sigma_{y}^{2}}-\frac{\tilde{y}_{0}^{2}}{2 \sigma_{y}^{2}}-\frac{x^{2}}{2 \sigma_{x}^{2}}-\frac{x_{0}^{2}}{2 \sigma_{x}^{2}}}}{(2 \pi)^{2} \sigma_{x}^{2} \sigma_{y}^{2}} d \tilde{y} d \tilde{x} d x_{0} d \tilde{y}_{0}
\end{gathered}
$$

where $n$ denotes a normalization factor, equal to the same quadruple integral without the factor in angular brackets, and $y_{\mathrm{co}}$ is a possible offset of the closed orbit from the centre of the two collimator jaws. In the actual experiment, the collimator was a horizontal one, for which we get the tune shift by replacing in the above expression all $x$ 's by $y$ 's and vice versa. The resulting theoretical prediction is also included in Fig. 4, using the emittances, intensities and bunch lengths measured at each point. Measurement and prediction agree within the uncertainties of the experiment.

\section{OBSERVATION OF TRAPPED MODES}

In addition to the main, broad band resistive wall collimator impedance, we also expect several, narrow impedance peaks, from the collimator, the surrounding tank and vacuum transitions. These will result in longer term wakes or "trapped modes". The trapped modes are not expected to be a problem for the beam stability, but could be an issue for local heating and relevant for cooling considerations. Calculations have been performed and trapped modes were measured with the beam.

Figure 5 shows a comparison of calculated and measured spectra, both for open and closed collimator settings. The most prominent modes, like for example the peak predicted at about $1.3 \mathrm{GHz}$, are clearly visible with the beam for closed collimators.

\section{CONCLUSION}

The LHC will have to be operated with many, relatively long carbon collimators. They are expected to dominate the transverse impedance and potentially limit the maximum stable beam intensity.

A prototype collimator has been installed in the SPS. Very small, but significant tune shifts were recorded as function of collimator settings with independent instruments and excellent agreement between the instruments. These tune shifts are directly related to the imaginary part of the transverse collimator impedance. The observed tune shifts agree well with the predictions for larger collimator settings. For small gap width corresponding to about $2 \sigma$,
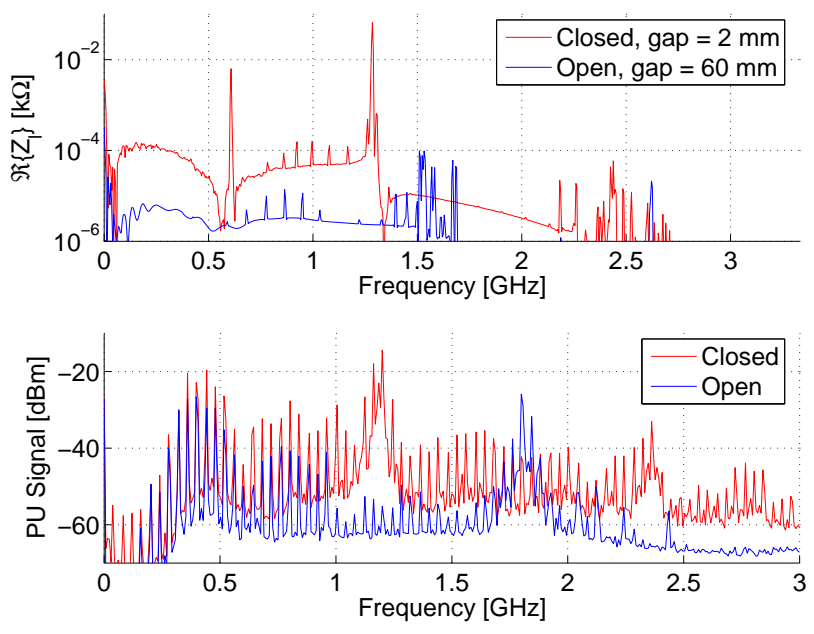

Figure 5: Calculated longitudinal modes (top) and longitudinal spectrum measured with the beam in the SPS (bottom), for open (blue) and closed (red) collimators.

the measured tune shifts are smaller than predicted by the linear model. A more detailed model which includes beam size effects and non-linear wakes is being studied.

Trapped modes have also been observed and the main modes match with expectations.

The measurements described here have already been very useful to check predictions and considerably increased our confidence in beam stability predictions for the LHC. We are working on more detailed predictions including non-linear wake and beam size effects and plan further measurements, possibly with multiple bunches, when the SPS will resume operation in the year 2006.

\section{REFERENCES}

[1] “The LHC design report, Vol.I Chapter 18." CERN-2004-003.

[2] A. Koschik, F. Caspers, E. Metral, L. Vos, and B. Zotter,Transverse resistive wall impedance and wake function with 'inductive bypass', Proc. EPAC 2004

[3] R. Assmann et al.,LHC Collimation: Design and Results from Prototyping and Beam Tests, this conference.

[4] F. Sacherer, Single-beam collective phenomena, Transverse, Bunched Beams, Proceedings of the 1976 Erice school on accelerators, M.H. Blewett (Ed.), CERN Yellow Report 77-13, (1977) p. $198 \mathrm{ff}$.

[5] M. Gasior and R. Jones, High Sensitivity Tune Measurement by Direct Diode Detection, To be presented at DIPAC 2005, Lyon, France, June 6-8 2005.

[6] K. Bane, J. Irwin, and T. Raubenheimer, Zeroth Order Design Report for the NLC, Vol. 2, p. 594 (1996).

[7] A. Piwinski, Impedances in Lossy Elliptical Vacuum Chambers, DESY-94-068 (1994). 